\title{
A Two-Stage Fuzzy Piecewise Logistic Model for Two Different Harmonic Excitations
}

\author{
Ruan Miao \\ School of Automobile Studies, Tongji University, Shanghai, Peoples Republic of China
}

\begin{abstract}
(Received 19 November 2012; revised 28 February 2013; accepted 25 April 2013)
\end{abstract}
It is very important for a firm to be able to forecast the sales volumes of new products. However, current environments usually have uncertain factors and rapid changes, and decision makers must make forecasts in these fluctuating situations with minimal data. Previous studies combine scenario analysis and the technology substitution model to forecast the market share of multigenerational technologies, which is one kind of interpolation method. However, a technology substitution model based on a logistic curve does not always fit the S-curve well. Therefore, based on historical data and the data forecasted by the scenario and Delphi methods, a two-stage fuzzy piecewise logistic growth model is proposed. The piecewise concept is adopted in order to understand the market impact of a new product so that it will be possible to determine the effective length of sales forecasting intervals even when handling large-variation data. In order to demonstrate the model's performance, the researchers used Tseng et al.'s data from 2009, the market share of an organic light-emitting diode (OLED) television (TV), and four other TV technologies in three possible scenarios of the global market: optimistic, pessimistic, and most possible. The results show that the proposed model outperforms the technology substitution model.

\section{INTRODUCTION}

The replacement of an older generation of technological products with newer ones is common in the high-technology sector. To better understand and describe this phenomenon, Fisher and Pry proposed the technological substitution model in 1971, which analyses the penetration process of newgeneration technologies as they replace old ones. ${ }^{1}$ Marchetti and Nakicenovic expanded this model to deal with the multigeneration and multi-products conditions. ${ }^{1,2}$ Nakicenovic has given a more comprehensive description of the model and its assumptions. ${ }^{3}$ These substitution models are market-share models that assume that there is a market to be substituted. From the innovation diffusion perspective, Norton and Bass developed a multi-generation diffusion model that has the ability to estimate or forecast a market potential and has become a popular forecasting method in the field. ${ }^{4}$

A key proposal of Marchetti and Nakicenovic is that the life cycle of every technology includes three phases: growth, saturation, and decline. ${ }^{2}$ During the growth phase of a new technology, substitution proceeds logistically. Then, when the technology enters the decline phase, it degenerates logistically. However, in the recent competitive environment, it is hard for the product life cycle to be represented by a smooth S-curve or a fitted-logistic curve. This is shown by Tseng et al., who proposed scenarios for the development of the organic light-emitting diode (OLED), a TV technology with no sales data; they proposed three scenarios: optimistic, pessimistic, and most possible. They then applied the Marchetti and Nakicenovic technological substitution model, in which the diffusion curves of all technologies are supposed to be logistic and are supposed to forecast the market share of the colour cathode ray tube (CRT), rear projection (RP), plasma display panel (PDP), liquid crystal display (LCD), and OLED TVs. ${ }^{2}$ They found that the diffusion curve of the PDP TV was not a logistic curve, but an S-curve, which does not meet the assumption of the Marchetti and Nakicenovic technological substitution model. ${ }^{2}$ Therefore, the forecasting performance was not quite good (Please refer to the MAPE of PDP TV in Tables 1-3).
In addition to the diffusion curve of new products, the existence of uncertainty in the environment also requires consideration. To handle the uncertainty issue, Tanaka et al. developed fuzzy regression with symmetrical-triangular fuzzy parameters via a possibility distribution. ${ }^{6}$ However, the conventional fuzzy regression model is sensitive to outliers. That is, the more the various data fluctuates, the wider the forecasted intervals become. These wide intervals then cause difficulties in interpretation. ${ }^{7}$ In order to cope with large variations in data, Yu et al. proposed the fuzzy piecewise regreson model, whose functions behave differently in different parts of the range of crisp input variables. ${ }^{8}$ Huang and Tzeng applied this model in their two-stage fuzzy piecewise regression analysis to grasp the dynamics of a nonlinear time series in a fuzzy environment. ${ }^{9}$ Their objective was to forecast product life time (PLT) and the annual shipment of products during the entire product life cycle (PLC) of multiple generation products. However, the number of change points could not be generated automatically. Yu and Tzeg extended the fuzzy piecewise regression model to overcome this limitation. ${ }^{10}$ A fuzzy piecewise logistic growth model combines the advantages of a piecewise regression model and the logistic diffusion model so as to deal with complex growth for single- or multiple- generation(s) product sales forecasting. ${ }^{11}$ This model can solve the problem that arises when the time series shows an S-curve but not a logistic curve, and it can be applied to multiple-generation diffusion forecasting. Hence, it has some applicability to real-world forecsting problems. However, the relative lack of data on the latest-generation products makes this model unsuitable for use in cutting-edge technology because the fuzzy regression model is limited to making forecasts inside the range of independent variables.

Scenario analysis, an important forecasting method since the 1960s, is another approach commonly employed to forecast and understand future developments. The approach outlines some aspects of the future world by telling stories to emphasize dimensions of today's world and to incorporate uncertainties. ${ }^{12}$ Scenario analysis thus presents rich and complex portraits of possible future scenarios; however, it fails to provide quantifi- 